\title{
Use of Glucose Rate of Change Arrows to Adjust Insulin Therapy Among Individuals with Type 1 Diabetes Who Use Continuous Glucose Monitoring
}

\author{
Jeremy Pettus, MD, and Steven V. Edelman, $\mathrm{MD}^{1,2}$
}

\begin{abstract}
Objective: This study was performed to understand and to compare differences in utilization of continuous glucose monitoring (CGM) and the rate of change (ROC) arrow to adjust insulin therapy among individuals with type 1 diabetes (T1D), comparing those treated with multiple daily insulin injections (MDI) with those treated with continuous subcutaneous insulin infusion (CSII).

Research Design and Methods: We surveyed 222 T1D individuals who regularly used real-time CGM to obtain information about general CGM use and response to glucose ROC arrows in managing their diabetes.

Results: The survey was completed by 222 T1D individuals. Respondents included CSII $(n=166)$ and MDI $(n=56)$ users. MDI and CSII respondents reported similar substantial increases in correction dosages (from $220 \mathrm{mg} / \mathrm{dL}$ to $120 \mathrm{mg} / \mathrm{dL}$ ) in response to increasing glucose (one ROC arrow up: rising $2-3 \mathrm{mg} / \mathrm{dL} / \mathrm{min}$ ): $+120 \%$ and $+108 \%$, respectively $(P=0.13)$. MDI and CSII respondents reported similar substantial increases in correction dosages in response to rapidly increasing glucose (two arrows up: rising $>3 \mathrm{mg} / \mathrm{dL} / \mathrm{min}$ ): $+146 \%$ and $+138 \%$, respectively $(P=0.72)$. When correcting from $220 \mathrm{mg} / \mathrm{dL}$ to $120 \mathrm{mg} / \mathrm{dL}$, MDI respondents reported larger correction dosage reductions than CSII respondents in response to decreasing glucose (one ROC down arrow: decreasing $2-3 \mathrm{mg} / \mathrm{dL} / \mathrm{min}$ ) and rapidly decreasing glucose (two ROC down arrows: decreasing $>3 \mathrm{mg}$ / $\mathrm{dL} / \mathrm{min}):-50 \%$ versus $-37 \%$, respectively $(P=0.024)$ and $-52 \%$ versus $38 \%$, respectively $(P=0.034)$. Similar between-group differences were observed in mealtime dosage adjustments.

Conclusions: CGM users often rely on ROC information when determining insulin doses and tend to make larger changes than current recommendations suggest regardless of insulin delivery method.
\end{abstract}

\section{Introduction}

$\mathbf{N}$ UMEROUS STUDIES HAVE DEMONSTRATED that utilization of real-time continuous glucose monitoring (CGM) improves glycemic control in individuals with type 1 diabetes (T1D) ${ }^{1-7}$ and is becoming accepted as part of the standard of care in the treatment of patients within this population. ${ }^{8,9}$ Use of real-time CGM provides similar benefits in glucose control for patients using multiple daily insulin injections (MDI) therapy ${ }^{5,6}$ and continuous subcutaneous insulin infusion (CSII) therapy. ${ }^{1-4}$ However, little is known about how patients utilize CGM data to adjust their insulin therapy on a day-to-day basis and if there are differences between individuals who use MDI therapy and those who utilize CSII.
In 2013, we surveyed 300 individuals with T1D $(n=222)$ and insulin-treated type 2 diabetes $(n=78)$ to assess how they are using real-time CGM data and responding to their glucose information in real-world settings. Our subsequent report presented findings regarding CGM data utilization behaviors among the T1D individuals who used real-time CGM and responded to the survey. ${ }^{10}$ In our primary analysis we found that respondents utilize the CGM data to alter multiple aspects of their diabetes care, including insulin dose timing, dose adjustments, and in hypoglycemia prevention. Moreover, the insulin adjustments reported by respondents were much larger than previously recommended. ${ }^{1,11,12}$ For this report we conducted a more extensive analysis of the dataset to determine if there are differences in use of real-time CGM

\footnotetext{
${ }^{1}$ University of California San Diego, San Diego, California.
}

${ }^{2}$ Veterans Affairs Medical Center, San Diego, California. 
data according to insulin treatment (MDI vs. CSII) among T1D respondents.

\section{Materials and Methods}

\section{Study design}

This national survey assessed data utilization behaviors and clinical outcomes among individuals with T1D who were currently using real-time CGM as part of their diabetes management regimen. The online survey was available between May 28 to August 26, 2013, using SurveyGizmo (Boulder, CO) and included 70 multiple-choice questions. An institutional review board waiver was obtained.

\section{Subjects}

Clinical endocrinologists and diabetes educators who actively prescribe CGM from across the United States were asked to recruit patients from their practices. Inclusion criteria were as follows: T1D and use regular use (average $\geq 6$ days/week) of the Dexcom CGM system (Dexcom, Inc., San Diego, CA). Individuals who agreed to participate in the study were provided a Web link to the survey.

\section{Survey instrument}

The survey comprised six sections: (1) patient characteristics; (2) general CGM use; (3) hypoglycemia prevention and management; (4) hyperglycemia prevention and management; (5) insulin dosing adjustments (both for incidental hyperglycemia not at meals and at mealtimes); and (6) realtime use versus retrospective analysis. In order to contextualize the information, many of the survey questions were framed as clinical scenarios that would be commonly experienced by patients either on MDI or using an insulin pump.

For correction insulin adjustments, respondents were provided a scenario in which it had been $4 \mathrm{~h}$ since taking any insulin or eating and their CGM device showed a glucose value of $220 \mathrm{mg} / \mathrm{dL}$ (confirmed by self-monitored blood glucose with a horizontal rate of change [ROC] arrow (less than $1 \mathrm{mg} / \mathrm{dL} / \mathrm{min}$ change), one or two up ROC arrows and one or two down ROC arrows (Fig. 1A).

For mealtime insulin adjustments, respondents were provided a scenario in which their CGM device showed a glucose value of $110 \mathrm{mg} / \mathrm{dL}$ and they were planning to eat $50 \mathrm{~g}$ of carbohydrates. They were asked how much insulin they would take for that meal when the trend arrow showed two up ROC arrows and two down ROC arrows (Fig. 1B).

The questions were beta-tested in 20 experienced CGM users and refined repeatedly to assure the questions were well understood, clear, and unambiguous. Based on beta testing, it was estimated to take $20-30 \mathrm{~min}$ to complete the survey. Respondents chose from one of three different surveys in which the questions were identical but the order of the sections varied. The survey instrument has published previously. ${ }^{10}$ Respondents received a $\$ 30$ gift card for completing the survey.

\section{CGM system}

The Dexcom CGM system includes a 7-day transcutaneous sensor, a transmitter, and a receiver for 7-day wearing periods. The system measures interstitial glucose every $5 \mathrm{~min}$ and displays the numerical value and glucose trend line in the hand-held receiver. Users can set audible alerts for current or impending hypoglycemia and hyperglycemia. ROC arrows indicate the direction and velocity of changing glucose levels. In this analysis, the focus was on one arrow (up or down) and two arrows (up or down) compared with typical dosages when glucose is static (flat arrow) (Fig. 2).

\section{Measures}

We assessed real-time CGM utilization behaviors among individuals with T1D, comparing those treated with MDI versus CSII therapy. Study measures included the following: general CGM use; overall response to CGM; and use of ROC arrows to adjust insulin therapy.

\section{Statistical methods}

The survey is descriptive; no hypothesis testing was performed, and no comparative analyses are made. Categorical variables are summarized using counts and percentages. Summary statistics for continuous variables are summarized using mean and SD values. Histograms and other graphical displays are used to illustrate the distribution of the survey responses. SAS version 9.3 or later software (SAS Institute, Cary, NC) was used to conduct data conversion and analysis.

\section{Results}

\section{Patient characteristics}

In total, 222 individuals with T1D from 22 states across the United States completed the survey. Among respondents, the mean age for CSII respondents was $45 \pm 14$ years with a duration of diabetes of $22 \pm 13$ years, $51 \%$ were male, $166(75 \%)$ used CSII, 56 (25\%) used MDI, and the self-reported hemoglobin A1c (HbA1c) level was $6.8 \pm 0.8 \%$. The mean age for MDI respondents was $48 \pm 13$ years with a duration of diabetes of $20 \pm 14$ years, $39 \%$ were male, and the self-reported $\mathrm{HbAlc}$ level was $6.8 \pm 0.9 \%$. No significant between-group differences were reported in age, gender, $\mathrm{HbAlc}$ level, or duration of diabetes. Most respondents (97\% CSII, 98\% MDI) reported having additional education after high school.

\section{General real-time CGM use}

Similar percentages of MDI and CSII respondents reported constant use of CGM: $85 \%$ versus $82 \%$, respectively $(P=0.3645)$. Respondents could choose from the following answers: "mainly when I see my control is slipping," "mainly just before I see my clinician," "most of the time," "all of the time," or "other." Among MDI respondents, 68\% reported wearing their CGM "all of the time," and 14\% reported wearing their CGM device "most of the time." Among CSII respondents, 65\% reported wearing their CGM device "all of the time," and 20\% reported wearing their CGM device "most of the time." Most MDI and CSII respondents reported using their CGM device for $>1$ year: $79 \%$ versus $74 \%$, respectively $(P=0.94)$.

\section{Overall response to CGM}

Most MDI (75\%) and CSII (79\%) respondents reported that the frequency and severity of hypoglycemia had decreased subsequent to starting CGM. Respondents could choose from 
A Correction dosage adjustment
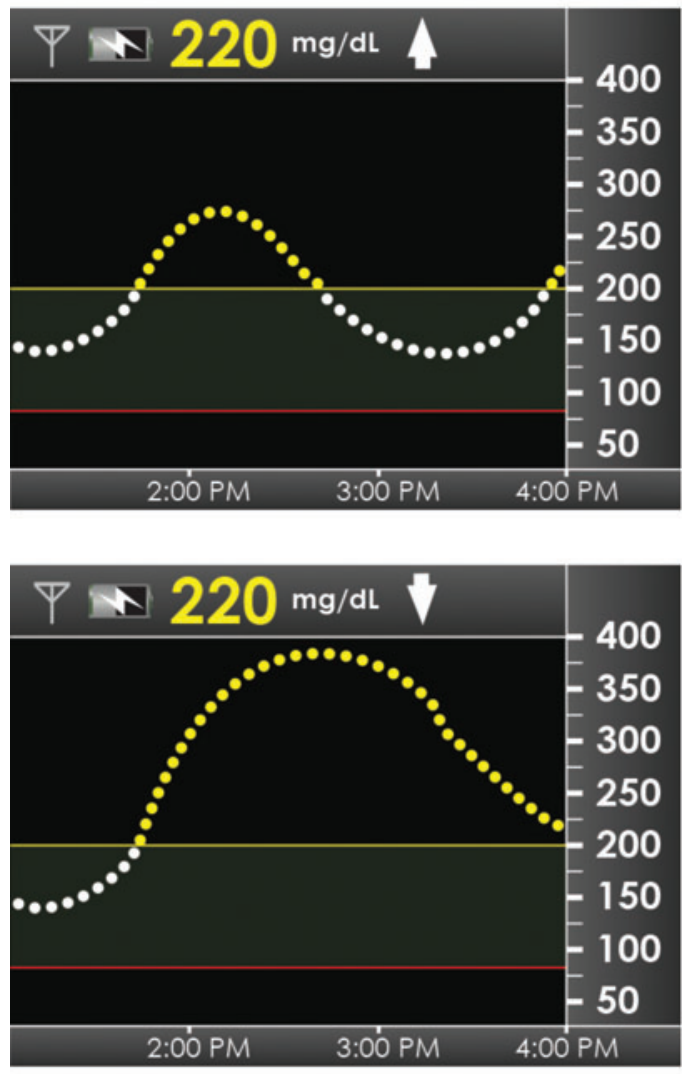

B Mealtime dosage adjustment

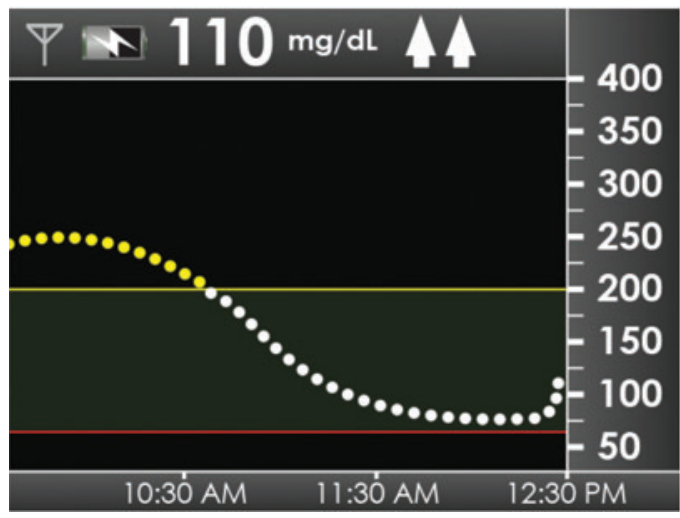

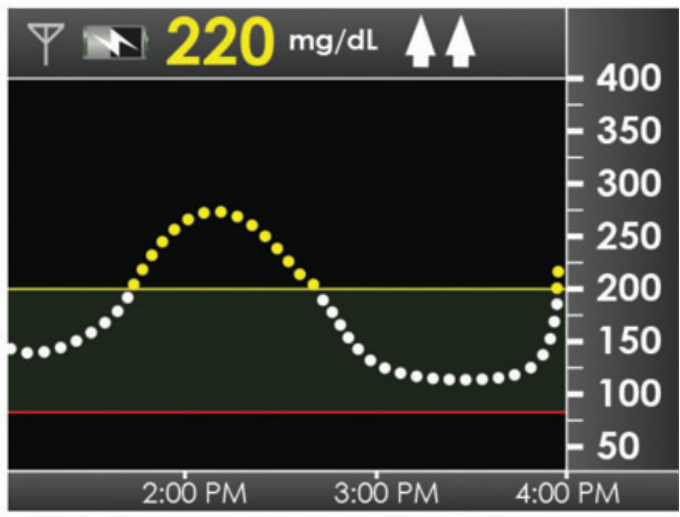
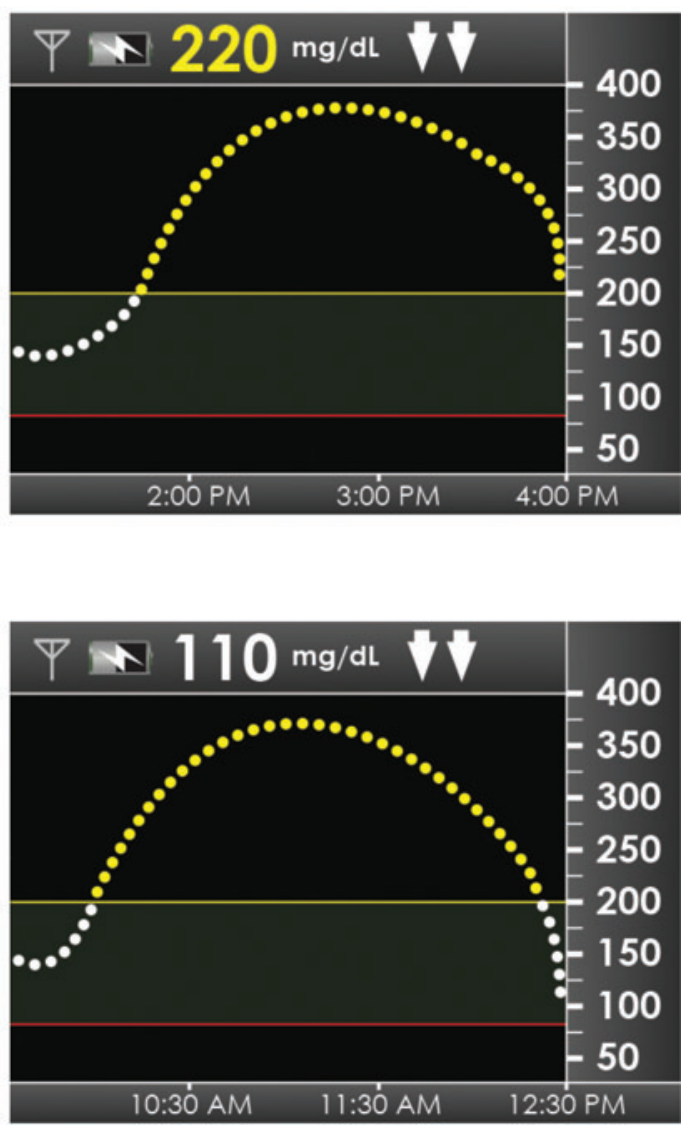

FIG. 1. (A) Scenarios for correction to $120 \mathrm{mg} / \mathrm{dL}$ in which respondents showed current glucose at $220 \mathrm{mg} / \mathrm{dL}$ but changing (one arrow up/one arrow down) or rapidly changing (two arrows up/two arrows down). (B) Scenarios in which respondents' continuous glucose monitoring device showed a glucose value of $110 \mathrm{mg} / \mathrm{dL}$ and they were planning to eat $50 \mathrm{~g}$ of carbohydrates (two arrows up/two arrows down).

the following answers: "increased a lot," "increased a little," "neither increased nor decreased," "decreased a little," or "decreased a lot." Similar percentages of MDI and CSII respondents reported that the frequency and severity "decreased a lot": $39 \%$ versus $42 \%$, respectively $(P=0.544)$. Most MDI and CSII respondents reported clinically significant decreases in HbA1c level since starting CGM: 39\% of MDI respondents reported HbA1c reductions of $0.5-1.0 \%$, and $16 \%$ reported reductions of $>1.0 \%$, whereas $33 \%$ of CSII respondents reported $\mathrm{HbA} 1 \mathrm{c}$ reductions of $0.5-1.0 \%$, and
$28 \%$ reported reductions of $>1.0 \%$. The between-group difference was not significant $(P=0.2178)$.

\section{Changes in injection/bolus frequency}

Most MDI (53\%) and CSII $(60 \%)$ respondents reported that the number of daily injections or boluses had increased (two or more additional) since starting CGM. A significantly larger percentage of MDI than CSII respondents reported that they were taking fewer daily injections or boluses: $37 \%$ 
FIG. 2. Rate of change arrows indicate the direction and velocity of changing glucose.

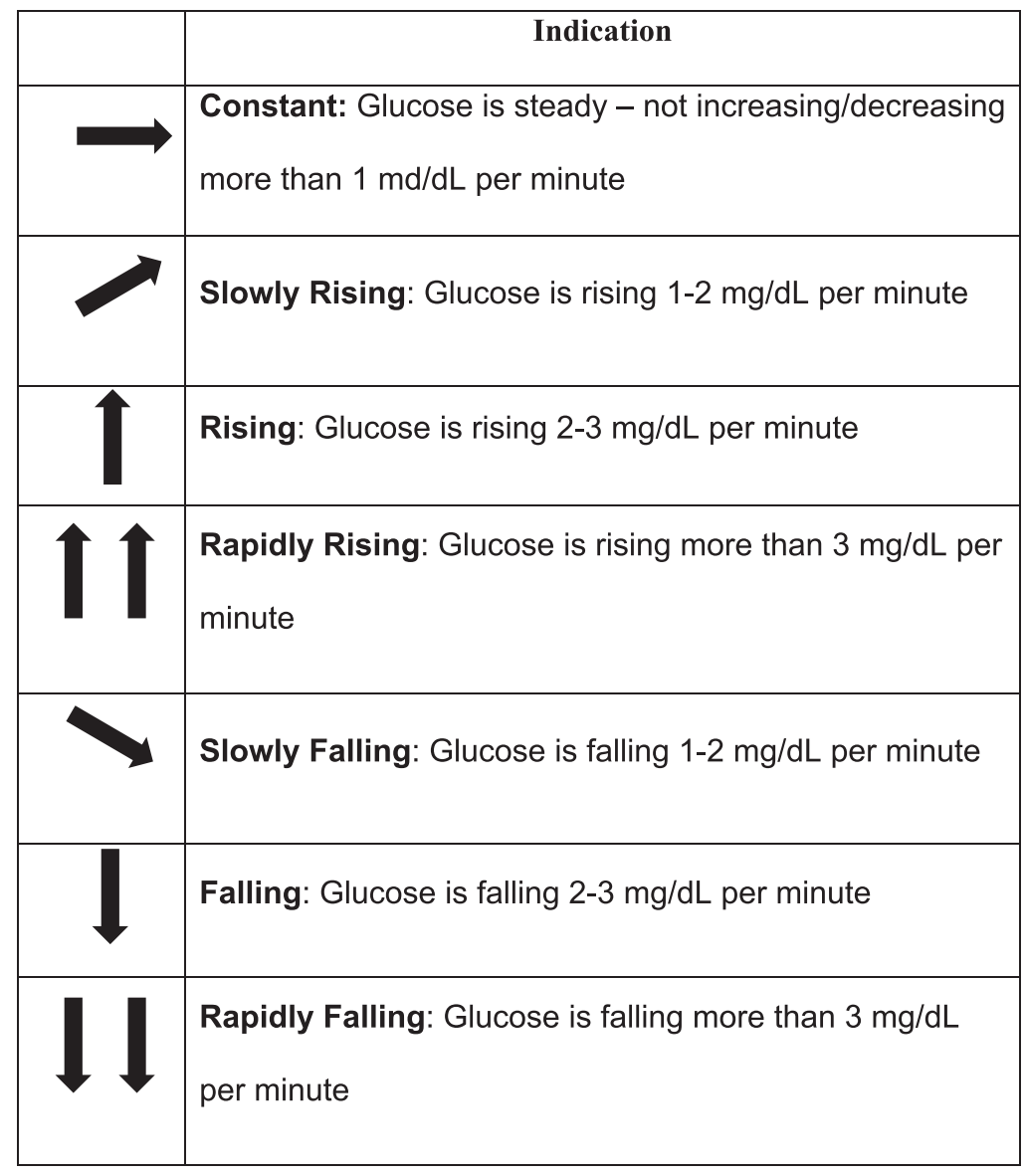

versus $18 \%$, respectively $(P<0.001)$. The remainder indicated no change or were not sure.

\section{Use of ROC arrows to adjust insulin therapy}

Determining insulin adjustments. A similar percentage of MDI (48\%) and CSII (53\%) respondents reported determining their insulin adjustments based on the CGM data by estimating from their previous experiences; however, a larger percentage of MDI $(30 \%)$ than CSII $(16 \%)$ respondents reported taking a fixed dose of insulin in response to increasing glucose $(P<0.0195)$.

Use of arrows: correction dosages. MDI and CSII respondents reported similar substantial increases in correction dosages in response to increasing glucose (one arrow up) compared with their typical dosage when glucose is stable (flat arrow) at $220 \mathrm{mg} / \mathrm{dL}$ in order to correct to $120 \mathrm{mg} / \mathrm{dL}$ : $+120 \%$ and $+108 \%$, respectively $(P=0.13)$ (Fig. 3A). MDI and CSII respondents also reported similar substantial increases in correction dosages in response to rapidly increasing glucose (two arrows up): +146 and $+138 \%$, respectively $(P=0.72)$ (Fig. 3B). MDI respondents reported making larger correction dosage reductions than CSII respondents in response to decreasing glucose (one arrow down) compared with their typical correctional dose when glucose is stable (flat arrow): $-50 \%$ versus $-37 \%$, respectively $(P=0.024)$ (Fig. 4A). MDI respondents also reported making larger correction dosage reductions than CSII respondents in their typical correction dose in response to rapidly decreasing glucose (two arrows down): $-52 \%$ versus $-38 \%$, respectively $(P=0.034)$ (Fig. 4B).

Use of arrows: mealtime dosages. MDI respondents reported making larger increases in their mealtime dosage than CSII respondents in response to rapidly increasing glucose (two arrows up) compared with their typical mealtime dose when glucose is stable (flat arrow) at $110 \mathrm{mg} / \mathrm{dL}:+95 \%$ versus $+77 \%$, respectively $(P=0.013)$ (Fig. 5A). MDI respondents also reported making larger reductions in their typical mealtime dosage than CSII respondents in response to rapidly decreasing glucose (two arrows down) when glucose is stable (flat arrow) at $110 \mathrm{mg} / \mathrm{dL}:-55 \%$ versus $-44 \%$, respectively $(P=0.019)$ (Fig. 5B).

Timing of meal insulin dose. Most MDI (61\%) and CSII $(58 \%)$ respondents reported adjusting the timing of their mealtime insulin dose relative to the meal based on the ROC; meals were delayed following mealtime insulin administration when arrows indicated increasing glucose.

\section{Discussion}

Our survey showed that most respondents persistently used their CGM devices at frequencies associated with significant glycemic improvements in large clinical trials. ${ }^{3,6,13-17}$ After 1 or more years of CGM use, most respondents reported reductions in frequency and severity of hypoglycemia, improvements in $\mathrm{HbA} 1 \mathrm{c}$ levels, increased number of daily injections/boluses, and more aggressive correction and mealtime 


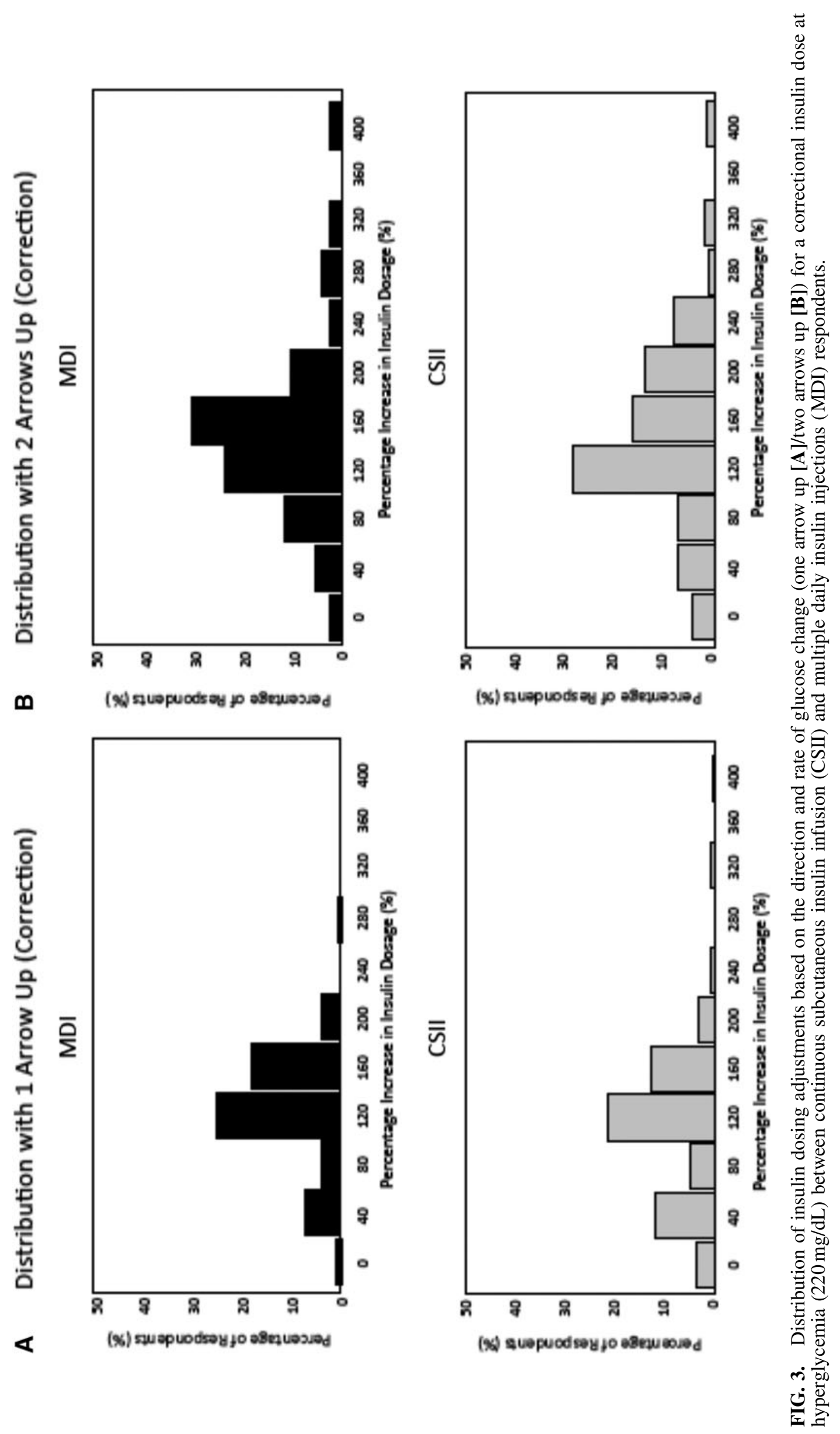




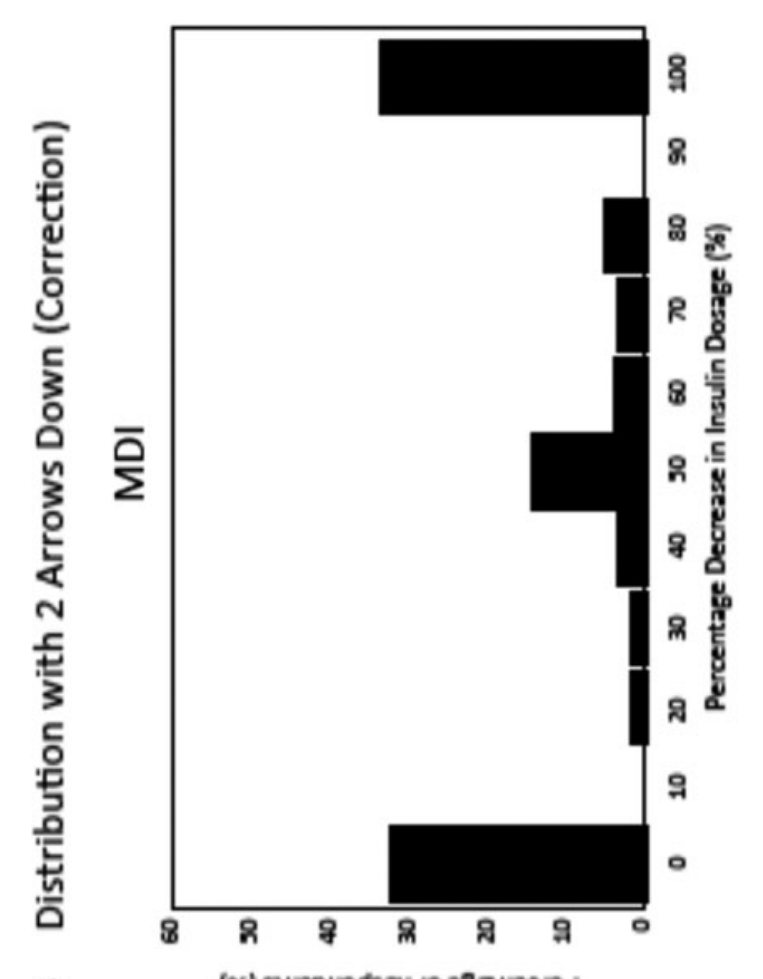

$\mathbf{m}$

(s) sauepuodsey pos a vesiod
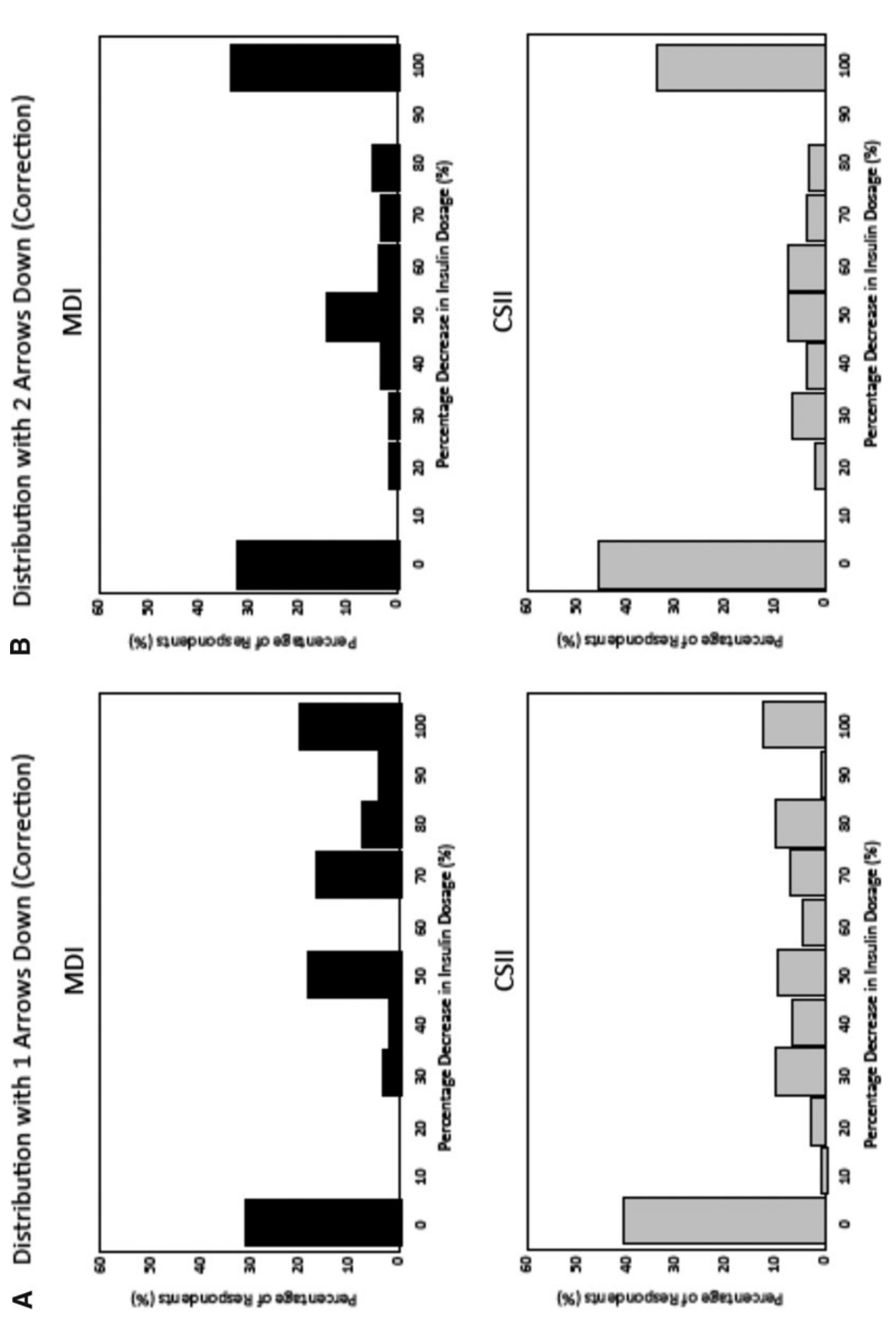

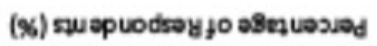
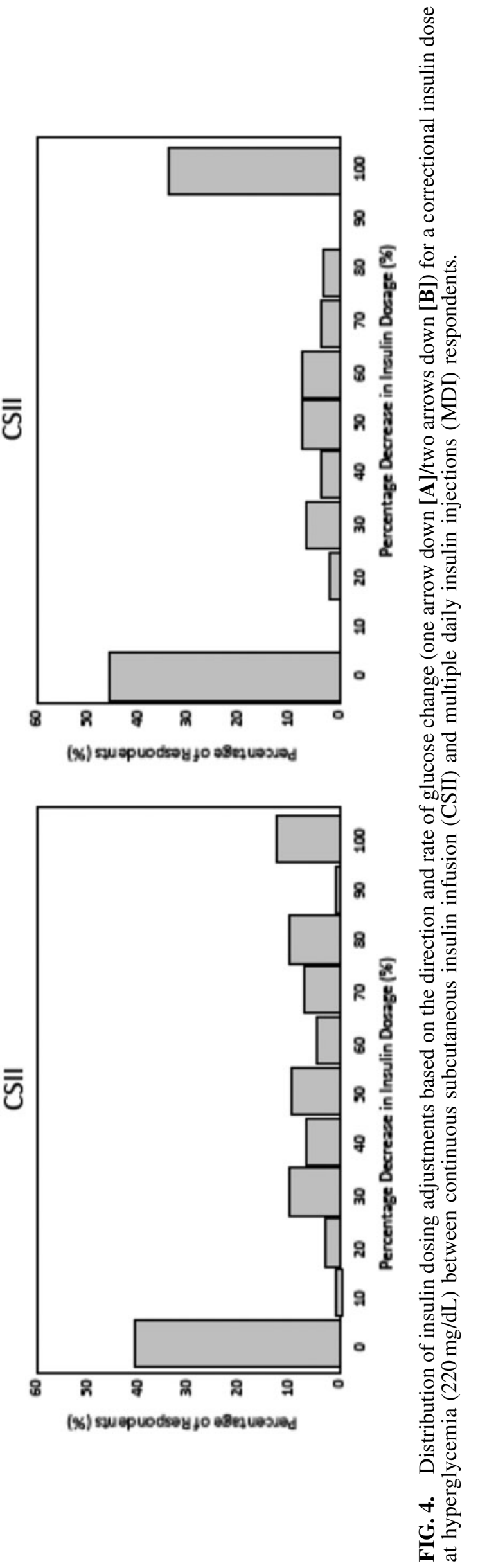


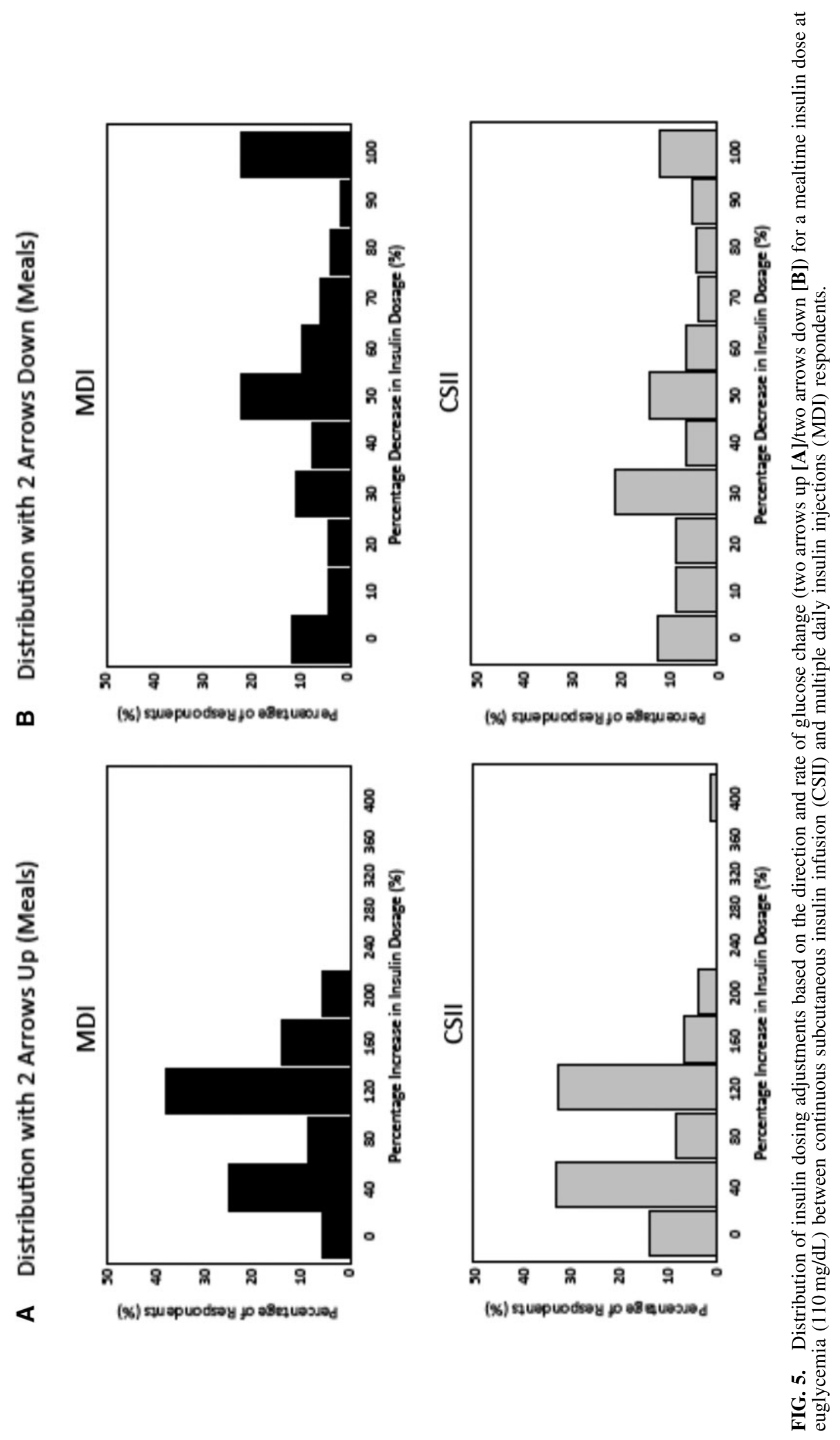


insulin dosage adjustments based on ROC arrows compared with the current recommendations.

Although most respondents reported an increase in the number of daily insulin injections/boluses, there were significant between-group differences. For example, a significantly larger percentage of MDI than CSII respondents reported taking fewer daily boluses. The ease of giving extra boluses and the ability to fine-tune insulin dose adjustments and decrease or suspend basal infusion with an insulin pump may explain this difference as has been demonstrated in an earlier study. ${ }^{2}$

It is also notable that a larger percentage of MDI than CSII respondents reported taking an extra fixed dose of insulin in response to rising glucose rather than considering past responses and adjusting based on experience or calculating (manually or via bolus calculator) a more exact dosage. Although this may be due to lack of confidence among MDI users regarding their dosage calculation accuracy, it is also possible that they felt confident that their CGM device would alert them to impending hyperglycemia or hypoglycemia if their fixed dose was inaccurate.

A key finding of our survey was the predominant use of ROC arrows and subsequent, what would be considered aggressive, correction and mealtime dosage adjustments among all respondents, regardless of insulin therapy. The majority of respondents reported using ROC arrows to make multiple and more significant changes in their insulin dosages than the $10-20 \%$ adjustments commonly recommended. ${ }^{1,11,12}$ A key issue is that although the ROC arrows were the driving factor in insulin dose adjustment, confidence in the accuracy of the glucose value was crucial. This suggests that respondents felt confident in the accuracy and reliability of their CGM device and their ability to closely monitor the effects of the dosage and take corrective action if needed.

Although both MDI and CSII users, on average, made larger than expected increases in their correction and mealtime dosages, MDI users made significantly larger adjustments in their mealtime dosages. This may be due to their desire to avoid taking additional correction injections following the meal. A possible explanation for the smaller reductions in both correction and mealtime insulin dosages among CSII respondents is that they can temporarily increase or decrease/suspend their basal rates as well as give additional smaller boluses of insulin over time.

A significant limitation of our survey was the use of selfreported data, which may not accurately reflect participants' actual frequency of CGM use or specific behaviors and/or dosage adjustments. Lack of objective measurements of clinical outcomes (e.g., change in $\mathrm{HbAlc}$, hypoglycemia frequency and severity) further limits the interpretation of our findings. Another limitation was use of a single CGM device brand (Dexcom), which limits the generalizability of our findings to other CGM systems. As demonstrated in an earlier study by Chamberlain et al., ${ }^{18}$ performance differences between CGM systems can impact users' perceptions of and trust in the accuracy and reliability of their CGM data. Our findings strongly suggest that user trust played a significant role in respondents' use of ROC arrows.

\section{Conclusions}

Findings from our survey demonstrated that many CGM users rely heavily on ROC arrows to make adjustments in their insulin dosages, regardless of their type of diabetes or insulin therapy. These adjustments are much larger than previously recommended and represent what people with T1D wearing real-time CGM are doing on a day-to-day basis. New guidelines are needed for insulin dose adjustments based on ROC in users with T1D.

\section{Acknowledgments}

The authors wish to thank Christopher G. Parkin, MS, for editorial support in developing this manuscript. Funding for the development of this manuscript was provided by Dexcom, Inc., San Diego, CA.

\section{Author Disclosure Statement}

J.P. has served as a consultant for Dexcom, Inc. S.V.E. has served as a consultant and advisory board member for and received research funding from Dexcom, Inc.

\section{References}

1. The Juvenile Diabetes Research Foundation Continuous Glucose Monitoring Study Group, Tamborlane WV, Beck RW, et al.: Continuous glucose monitoring and intensive treatment of type 1 diabetes. N Engl J Med 2008;359:1464-1476.

2. Battelino T, Conget I, Olsen B, et al.: The use and efficacy of continuous glucose monitoring in type 1 diabetes treated with insulin pump therapy: a randomised controlled trial. Diabetologia 2012;55:3155-3162.

3. Deiss D, Bolinder J, Riveline JP, et al.: Improved glycemic control in poorly controlled patients with type 1 diabetes using real-time continuous glucose monitoring. Diabetes Care 2006;29:2730-2732.

4. Bergenstal RM, Tamborlane WV, Ahmann A, et al.: Effectiveness of sensor-augmented insulin-pump therapy in type 1 diabetes. N Engl J Med 2010;363:311-320.

5. Tumminia A, Crimi S, Sciacca L, et al.: Efficacy of real-time continuous glucose monitoring on glycaemic control and glucose variability in type 1 diabetic patients treated with either insulin pumps or multiple insulin injection therapy: a randomized controlled crossover trial. Diabetes Metab Res Rev 2015;31:61-68.

6. Bailey TS, Zisser HC, Garg SK. Reduction in hemoglobin A1C with real-time continuous glucose monitoring: results from a 12-week observational study. Diabetes Technol Ther 2007;9:203-210.

7. Garg SK, Voelmle MK, Beatson CR, et al.: Use of continuous glucose monitoring in subjects with type 1 diabetes on multiple daily injections versus continuous subcutaneous insulin infusion therapy: a prospective 6-month study. Diabetes Care 2011;34:574-579.

8. Blevins TC, Bode BW, Garg SK, et al.: Statement by the American Association of Clinical Endocrinologists Consensus Panel on continuous glucose monitoring. Endocr Pract 2010;16:730-745.

9. Klonoff DC, Buckingham B, Christiansen JS, et al.: Continuous glucose monitoring: an Endocrine Society Clinical Practice Guideline. J Clin Endocrinol Metab 2011;96:29682979.

10. Pettus J, Price DA, Edelman SV: How patients with type 1 diabetes translate continuous glucose monitoring data into diabetes management decisions. Endocr Pract 2015;21: 613-620. 
11. Buckingham B, Xing D, Weinzimer S, et al.: Use of the DirecNet Applied Treatment Algorithm (DATA) for diabetes management with a real-time continuous glucose monitor (the FreeStyle Navigator). Pediatr Diabetes 2008;9:142-147.

12. Hirsch IB: Clinical review: Realistic expectations and practical use of continuous glucose monitoring for the endocrinologist. J Clin Endocrinol Metab 2009;94:2232-2238.

13. Effectiveness of continuous glucose monitoring in a clinical care environment: evidence from the Juvenile Diabetes Research Foundation continuous glucose monitoring (JDRFCGM) trial. Diabetes Care 2010;33:17-22.

14. Raccah D, Sulmont V, Reznik Y, et al.: Incremental value of continuous glucose monitoring when starting pump therapy in patients with poorly controlled type 1 diabetes: the RealTrend study. Diabetes Care 2009;32:2245-2250.

15. O'Connell MA, Donath S, O'Neal DN, et al.: Glycaemic impact of patient-led use of sensor-guided pump therapy in type 1 diabetes: a randomised controlled trial. Diabetologia 2009;52:1250-1257.
16. Battelino T, Phillip M, Bratina N, et al.: Effect of continuous glucose monitoring on hypoglycemia in type 1 diabetes. Diabetes Care 2011;34:795-800.

17. Frontino G, Bonfanti R, Scaramuzza A, et al.: Sensoraugmented pump therapy in very young children with type 1 diabetes: an efficacy and feasibility observational study. Diabetes Technol Ther 2012;14:762-764.

18. Chamberlain J, Dopita D, Gilgen E: Persistence of continuous glucose monitoring use in a community setting 1 year after purchase. Clin Diabetes 2013;31:106-109.

Address correspondence to: Steven V. Edelman, MD Veterans Affairs Medical Center San Diego, CA

E-mail: svedelman@vapop.ucsd.edu 